\title{
THE HIGH LATITUDE CLOUD LYNDS 1642 IS NOT BREAKING UP
}

\author{
T. Liljeström \\ Helsinki University Observatory \\ Tähtitorninmäki \\ SF-00130 Helsinki, Finland
}

\begin{abstract}
CO}, \mathrm{HCO}^{+}$and $\mathrm{NH}_{3}$ observations have been carried out towards the high latitude cloud L1642 using the 1.2-m GISS, the 11-m NRAO, the 14-m Metsähovi and the 100-m Effelsberg radio telescopes. The velocity field of the $C O$ gas indicates a core-halo structure. The core component has a constant radial velocity, whereas the halo gas is slightly $(<1$ $\mathrm{km} / \mathrm{s}$ ) redshifted, as compared to the radial velocity of the core, and shows velocity gradients towards the cloud edges in a similar way as the HI gas associated with L1642. Within the border of the ${ }^{13} \mathrm{CO}$ emission the mass of the cloud is estimated to be some $76 \mathrm{M}_{\odot}$. In contradiction to Magnani et al.(1985), who claimed that L1642 belongs to a population of very young high latitude clouds which are breaking up, this study supports the view that $\mathrm{L} 1642$ is in virial equilibrium and significantly older than $10^{6} \mathrm{yr}$. The virial equilibrium of $\mathrm{L} 1642$ enables a distance determination of $\sim 190 \mathrm{pc}$ to the cloud core.
\end{abstract}

\section{SIGNS OF SHOCK INDUCED CLOUD IMPLOSION IN L1642}

The Orion-Eridanus region contains large arc-like features, which clear1y imply that supernova explosions have disturbed the interstellar medium. Due to the lower density of the medium at high galactic latitudes, disturbances can propagate to much larger distances as compared to the galactic disk. Thus it is natural to expect that shock fronts from supernovae of the Orion I OB association have passed also the $190 \mathrm{pc}$ distant L1642 flattening the cloud in the direction of the arriving shock front and initiating a flow in the cloud envelope. It is also noteworthy that the extinction map of $\mathrm{L} 1642$ (Liljeström et al., 1988) shows pronounced dust "tongues" penetrating outwards from the cloud, a characteristic sign of Rayleigh-Taylor and Kelvin-Helmholtz instabilities.

These similarities with Woodward's model(1976) support an interpretation of a shock induced cloud implosion. Woodward's simulations predict also the formation of cloud condensations, some of which may be dense enough to undergo gravitational collapse. L1642 has produced two low-mass double stars. The more embedded one, associated with a co outflow, is located in the outer part of the core region in a "tongue" structure. 
This is the predicted geometry and location for a new star in the model of Woodward (1976) . The time for a shock driven low mass star formation is of the order $10^{7} \mathrm{yr}$ (Woodward, 1976) which is an order of magnitude longer than the dynamical time scale derived for high latitude clouds (Magnani et al., 1985).

\section{DYNAMICAL STATE OF L1642 AND CORE DISTANCE}

The virial theorem for a stationary cloud is $M\left(M_{\odot}\right)=c \sigma^{2} R_{p c}$, where $M$ is the cloud mass, $R$ the effective cloud radius, ${ }_{C}$ a constaht depending on cloud geometry and density structure, and $\sigma$ the 3 -dimensional velocity dispersion. The best distance estimate to $\mathrm{L} 1642$, based on uvby and $\mathrm{H}_{B}$ photometry (Franco, 1989), is $114<\mathrm{r}<230 \mathrm{pc}$. Adopting a mean value, $\mathrm{r} \underline{\underline{\beta}}$ $170 \mathrm{pc}$, the virial masses of $\mathrm{L} 1642$ range from 50 to $97 \mathrm{M}_{\odot}$ when 4 different cloud models are considered. Comparing these with the observed $M_{\text {to }}$ $=75 \mathrm{M}_{\odot}$ (for $\mathrm{r}=170 \mathrm{pc}$ ) it is obvious that $\mathrm{L} 1642$ is in virial equilibrium. The virial equilibrium of $\mathrm{L} 1642$ enables a distance determination to the cloud core. From the virial theorem one obtaines

$$
\sigma_{\text {vir }}(3-\operatorname{dim})=\sqrt{\mathrm{M}\left(\mathrm{M}_{\odot}\right) / \mathrm{C} \mathrm{R} \mathrm{pc}}=\sqrt{\mathrm{Ar}_{\mathrm{pc}}^{2} / \mathrm{C} \mathrm{Dr} \mathrm{pc}} \Leftrightarrow \mathrm{r}_{\mathrm{pc}}=\sigma^{2} \mathrm{C} \mathrm{D} / \mathrm{A}
$$

where $A$ and $D$ are the numerical coefficients of $r_{p c}^{2}$ and $r$ in the observed cloud mass and effective cloud radius, respectively, and $C$ the constant in the virial theorem. Substituting the numerical values obtained from the observations the most probable core distance of $190 \mathrm{pc}$ is obtained, which is in accordance with the results of Franco (1989).

The line widths of ${ }^{13} \mathrm{CO}$ and even ${ }^{12} \mathrm{CO}$ have been commonly used to derive the velocity dispersion of a cloud. However, in addition that these line widths have not been corrected for opacity broadening, their use causes an observational selection bias towards the higher velocity dispersions of the envelope gas, which do not necessarily reflect the gas motions which oppose the gravitational collapse of a cloud. Especially it should be stressed that if systematic gas motions are included into the kinetic energy of a cloud (as e.g. Magnani et a1. (1985) in L1642), the virial theorem of a bound and stationary system must be changed to

$$
(1 / t) \int_{0}^{t}(d / d t) \Sigma \vec{p}_{j} \cdot \vec{r}_{j}=2\left\langle E_{k i n}\right\rangle+\left\langle\Sigma \vec{F}_{j} \cdot \vec{r}_{j}\right\rangle
$$

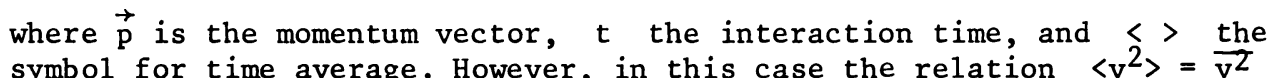
is not valid any more. Therefore, the velocity component of the halo gas should be examined carefully before it is included into virial theorem considerations.

\section{REFERENCES}

Franco, G.A.P. 1989, Astron. Astrophys. 223, 313 Liljeström, T. and Mattila, K. 1988, Astron. Astrophys. 196, 243 Magnani, L., B1itz, L. and Mundy, L. 1985, Astrophys. J. 295, 402 Woodward, P. 1976, Astrophys. J. 207, 484 